\title{
Important Differences in Accelerometer Cut Points for Quantifying Physical Activity in a Nested Occupational Cohort
}

\author{
Matthew S Thiese ${ }^{1 *}$, Kurt T Hegmann ${ }^{1}$, Timothy K Behrens ${ }^{2}$, Arun Garg ${ }^{3}$ and \\ Christina Porucznik ${ }^{1}$ \\ ${ }^{1}$ Department of Family and Preventive Medicine Rocky Mountain Center of Occupational and Environmental Health, University of Utah, Salt Lake City, Utah \\ ${ }^{2}$ Department of Health Sciences, University of Colorado Colorado Springs, Colorado Springs CO \\ ${ }^{3}$ Department of Industrial and Manufacturing Engineering, University of Wisconsin-Milwaukee, Milwaukee, WI
}

Received: November 20, 2013; Accepted: December 24, 2013; Published: J anuary 02, 2014

${ }^{*}$ Corresponding author: Matthew S Thiese, Department of Family and Preventive Medicine, University of Utah, 391 Chipeta Way Suite C, Salt Lake City, UT 84108, USA, Tel: 801-587-3322; E-mail: matt.thiese@hsc.utah.edu

\begin{abstract}
Background: Accurate physical activity assessment is becoming increasingly important in epidemiologic research. There is an increasing body of evidence suggesting that physical activity can prevent or reduce severity of disease. Utilizing accelerometers as an objective quantification of physical activity is a cost effective way to assess physical activity, yet there is little research on the agreement between different proposed cut points in a free-living, healthy adult population.
\end{abstract}

Methods: This study investigated the correlation, sensitivity and specificity of different cut points for light, moderate, and vigorous activity in an occupational cohort of 78 participants. All participants wore the accelerometer for at least 12 hours a day for at least 5 days, including one weekend day.

Results: There was a broad range of minutes of light and moderate activity and few minutes of vigorous activity. In general, correlations between free-living derived cut points were lower than those comparing laboratory derived cut points. Sensitivity and specificity values covered a broad range of values with lower sensitivity values in the light and moderate activity level categories and lowest specificity values in the light activity category.

Conclusions: Cut points derived from laboratory studies are generally more congruous but may not be more accurate. There is little agreement between cut points at light and moderate activity levels. Consideration of the population being studied should be taken into account when deciding on what set of cut points to use.

\section{Abbreviations}

PA: Physical Acitvity; V02: Volume of Oxygen consumed; METs: Metabolic Equivalents

\section{Introduction}

Physical activity (PA) is an important element in everyday life. Many studies have reported statistical associations between PA and a variety of health outcomes [1-14]. These studies rely on various measures of PA to statistically evaluate associations. However, PA is multidimensional and when considering the measurement of PA, it may be important to measure different elements and combinations of PA (duration, intensity, frequency and type). Current recommendations are that a minimum of 30 minutes of moderate-intensity activity on most days of the week is required for good health $[14,15]$. It is possible that there may be differential health effects of different durations, intensities, frequencies and patterns of PA than those suggested, depending on the outcome affected.

Many objective tools may be utilized to measure PA, including direct measurement using doubly-labeled water or direct observation, pedometers and accelerometers, or questionnaires. Doubly-labeled water and direct observation are expensive and obtrusive and are not normally used in population research, whereas questionnaire data are inexpensive, but chronically misreported. The use of accelerometer or pedometer motion sensors allow for objective and reliable measurement of ambulatory PA [16]. Accelerometers are instruments for the assessment of dynamic activities and are generally considered to be the most accurate objective measure of ambulatory activity short of direct observation. Their use is recommended in combination with activity diaries to enable information to be collected on the type of PA and domain of activity [17]

There have been multiple studies assessing the appropriateness of cut points for counts per minute assessed by accelerometer measures for data reduction. The first and most widely used was performed by Freedson [18]. Other published articles propose cut points of varying levels including articles [1927]. The goal of this study is to report similarities and differences between the various cut points by applying them to a study population, and stress the importance of selecting the correct cut points for the population and type of activity being studied. The proper selection of cut points is important for epidemiologic research as well as for public health policy. For example, if a study was assessing physical activity only as controlled walking on a flat surface, the cut points would be very different than free-living physical activity assessment in a manufacturing facility during 
a work shift. The cut points selected may be dependent upon the population and range of potential physical activities being assessed. This study is designed to demonstrate the variability and agreement in physical activity levels when applying different commonly used cut points on a free-living population.

\section{Methods}

\section{Participants}

Participants were selected from an ongoing prospective cohort study investigating potential occupational and nonoccupational risk factors for low back pain. Additional study details are described elsewhere [28-30]. Selection criteria for participants in the original cohort included a stable workforce with no anticipated changes in jobs; worker and management enthusiasm and cooperation for the study; consistent types of work; availability of jobs in low, medium, and high job physical factor exposure groups; and gender representation (i.e., populations of both males and females). All participants enrolled in the ongoing cohort study at these three plants in Utah were eligible to participate in the current study. Of 119 invited to participate, 14 declined to wear the accelerometer and 27 wore the accelerometer but did not meet the a priori standards for accelerometer data of 12 or more hours each day for at least 5 days, including one weekend day. The resulting 78 participants' data were analyzed.

\section{Ethics Statement}

This research has been approved by the University of Utah Institutional Review Board (IRB \# 11889) and all participants provided written consent prior to participating in research.

\section{Accelerometers}

Participants $(n=105)$ wore the Actigraph accelerometers model GT1M (Actigraph LLC) monitor on their right hip during waking hours for a minimum continuous 7-day study period duration. Participants were instructed not to wear the accelerometer during periods of bathing and sleeping; however the accelerometer was to be worn at all other times. Participants were allowed to remove the accelerometer if it caused discomfort or was at risk of damage. Participants were invited to participate if they had little or no exercise habits that would not be well captured by the accelerometer (e.g., nonambulatory activities such as lifting weights, swimming, etc.) All participants who agreed to wear the accelerometer were given verbal and written instructions on its use and importance immediately before commencing the 7-day study period.

The Actigraph is a small ( $2.0 \times 1.6 \times 0.6$ inches), light $(0.09$ pounds), unobtrusive uniaxial accelerometer. Epoch length was 10 seconds, which were summed to 1 minute epoch lengths, as is standard for field studies in adult populations [31]. The 10-second epoch length was selected to allow for increased resolution and the possible incorporation of additional calorimetric equations to estimate energy expenditure based on variation of each 10-second epoch within each minute [26]. Minute-by-minute data were summarized into daily averages for average (counts per minute per day) and total activity counts (counts per day) for times when the monitor was worn, and for activity durations (minutes per day) in the activity levels outlined by a variety of cut points.

Overall compliance rates for utilization of the Actigraph accelerometers was relatively high. Compliance was further increased by encouraging participants to complete a diary, showing participants examples of output to demonstrate how it measures activity, and by providing participants a list of frequently asked questions [31]. Other studies have reported high compliance rates utilizing some of these methods [31,32]. At the conclusion of the accelerometer data collection period a 7-day PA recall [33] was administered by the researcher to further assess activity components. This metric was designed to further capture details regarding the participants' PA levels while they were wearing the accelerometer. This assessment tool captures data for the time frame including participant rating of the past 7 days as compared to normal activity levels over the past 3 months and self-report any conditions (e.g., injury or illness) that may affect accelerometer accuracy with a subsequent description. PA diaries were also kept by participants for the time frame they were wearing the accelerometer and collected along with the accelerometer.

\section{Data reduction cut points used}

Freedson: The Freedson cut points [18] are based on a young volunteer participant population of 25 males and 25 females. Participants were asked to not exercise or ingest caffeine for 4 hours before the test. Participants performed 6 minutes on a motorized treadmill of 1 ) slow walking (4.8 kilometers per hour), 2) fast walking (6.4 kilometers per hour), and 3) jogging (9.7 kilometers per hour) for a total of 24 minutes of activity with verified treadmill speed. Each 6-minute activity bout was separated by a 5-minute rest period and the order of activities was even across participants. Oxygen consumption was measured per minute using spirometry with computer-based data collection. The analyzers were calibrated before each test using verified gases of known concentration. Steady-state bounds were the average the final 3 minutes (minutes 4-6) of exercise for each activity. METs were calculated using the standard calculation of dividing the steady-state $\cdot \mathrm{VO} 2$ by 3.5 milliliters per kilogram per minute. The uniaxial accelerometer used is the Computer Science and Applications (CSA) Model 7164 with parameters that detect typical body movement and filter out high frequency movement such as vibration. It was secured to the right hip via belt. Each digitized signal is summed over a user specified time interval (epoch), using a 60-second epoch with activity counts expressed as the mean counts per minute over the 6 minutes of activity. One-way repeated measures ANOVA and linear regression were used to assess intensity for all dependent variables and to assess the relationship between metabolic cost and counts. Multiple linear regression was used to estimate caloric expenditure. There were no differences between males and females reported. A one-way repeated measures ANOVA revealed a statistically significant relationship for all dependent variables. The relationship is linear $(r=0.88)$. Freedson concludes that there 
is adequate discrimination between count ranges to discern different intensities of exercise. These data suggest that 1258 counts corresponds to a 1 MET change between 3 and 9 METs using 1951 counts as the minimum to define the 3 MET level (considered moderate activity). The hard (vigorous) activity cut point is $\mathbf{5 7 2 5}$ counts per minute and very hard cut point is 9499 counts per minute.

Hendelman: Ten male and 15 female volunteers between the ages of 30 and 50 participated in the study and completed a PA readiness questionnaire prior testing. Participants participated in three test sessions, with a subset retesting for reliability. The first session consisted of walking at four self-selected speeds; "leisurely" (bout 1), "comfortable" (bout 2), "moderate" (bout 3 ), and "brisk" (bout 4) paces for approximately 5 minutes each, with 5 minutes of rest between bouts, on an indoor track. These verbal anchors were open to the individual participant's interpretation and were not quantified. Speed and step frequency were measured. The second session consisted of continuous play of two holes of golf with pull cart for their clubs. The final session involved household tasks consisting of 5 minutes each of washing windows, dusting, vacuuming, lawn mowing (using a gas-powered push mower), and planting shrubs. During all sessions, respiratory gas exchange was assessed using a portable metabolic measurement system carried in a pack on the lower back. In addition, three motion sensors were worn on elastic belts around the waist. A uniaxial CSA accelerometer model 7164, was worn on the right hip and a triaxial Tritrac monitor was worn on the left hip. Cardiorespiratory and accelerometer data were averaged over 1-minute epochs. Participants were to maintain similar diet and activity levels and all sessions were conducted at the same time of day for each subject. Body mass, food and caffeine intake, prior exercise and environmental conditions were recorded for each session. For the walking session, metabolic and accelerometer data were averaged over the final 2 minutes of each of the four speeds. For the other two activities, metabolic and accelerometer readings were averaged after the initial 2 minutes. All metabolic data were adjusted for the mass of the equipment worn.

Utilizing Pearson product moment correlation coefficients the authors assessed the relationships between metabolic cost and the count data from the two accelerometers 1) for the walking sessions only and 2) for all activities combined. Regression analysis used to develop equations predicting metabolic cost from activity counts with cutoff values resultant to preset MET levels (light activity, > 1 MET to < 3.0 METs; moderate activity, $>=3.0$ METs to <6.0 METs; and hard activity, $>=6.0$ METs to $<9.0$ METs). Cutoff values established from each equation (walking and all activities) were compared for both accelerometers. In addition, the CSA values were compared with previously reported cutoff values for these intensity levels.

The data from the four walking speeds were used to develop individual regression equations relating $\mathrm{VO}_{2}$ to CSA and Tritrac counts for each subject. These equations served as individualized "calibration curves" for the accelerometers. Subsequently, the count values from the golf and household activities were substituted into the equations to predict MET values from each accelerometer. These predictions were compared with the actual measured METs.

All analyses are based on the entire sample. Correlations between metabolic cost and accelerometer output were examined. For the walking trials only, the correlation between CSA counts and METs was relatively high $=0.77$ however, the correlation for all activities combined was lower $(r=0.59)$. Adequate reproducibility was seen in the count versus MET relationships, with similar regression equations and correlations observed for both trials for the subjects who performed reliability testing. For the CSA monitor, these correlations were $r=0.78$ (walking only) and $r=0.58$ (all activities) for trial 1 and $r=0.78$ and 0.65 for trial 2 .

The regression equations were used to determine the count values corresponding to the MET ranges defining light (1.0 MET to $<3.0 \mathrm{METs}$ ), moderate ( $>=3 \mathrm{METs}$ to $<6 \mathrm{METs}$ ), and hard ( $>=6$ METs to $<9$ METs) activity categories. The count values differed significantly for the walking data compared to all activities combined. For the walking data, light activity was defined as $<2191$, moderate was $<6893$, and hard was $\geq 6893$. Conversely, for all activities combined light activity was defined as $<191$, moderate was $<7526$, and hard was $\geq 7526$.

The accelerometers underrated the intensity of each of the activities (30.5-56.8\%) with statistically significant effects of activity $(\mathrm{F}=164.6, \mathrm{P}<0.001)$ and method of measurement (actual vs CSA prediction vs Tritrac prediction) for $\mathrm{VO}_{2}(\mathrm{~F}=168.7$, $\mathrm{P}<0.001)$ as well as a significant interaction effect ( $\mathrm{F}=20.3, \mathrm{P}<$ 0.001 ). The interaction indicates that there is a differential under prediction of $\mathrm{VO}_{2}$ depending on the different activities.

Swartz: Thirty one males (ages $41 \pm 17$ years, mean \pm SD) and 39 females (ages $42 \pm 14$ years, mean \pm SD) volunteer participants completed 15 minutes of one to six activities within one or more categories for a total of 5-12 participants tested per activity. The activities included:

- Yard work: mowing the lawn (manual and power mowers); raking; trimming (power trimmer or "weed-eater"); gardening (pulling weeds, planting flowers).

- Occupation: walking at 67 meters per minute and carrying items weighing 6.8 kilograms; walking at 93.8 meters per minute and carrying items weighing 6.8 kilograms; loading and unloading boxes weighing 6.8 kilograms.

- Housework: vacuuming; sweeping and mopping; laundry; ironing; washing dishes; cooking; light cleaning (dusting, general picking up); grocery shopping with a cart.

- Family care: feeding and grooming animals; caring for small children; playing with children in the yard; playing with animals in the yard.

- Conditioning: stretching; light calisthenics; slow walking (average speed 78 meters per minute); brisk walking (average speed 100 meters per minute). 
- Recreation: doubles tennis; golf in a two-some or four-some (carrying clubs); golf in a two-some or four-some (pulling clubs); softball.

Prior to each activity participants were asked to sit quietly for 5 minutes as a control period. The activities were performed in the exercise physiology laboratory (occupation, conditioning), within the university grounds (recreation), at the participant's home (yard work, housework, family care), and at a local golf course (golf) and tennis club (doubles tennis).

Each participant wore a portable indirect calorimetric system, while performing each activity and throughout the rest periods. Mean $\mathrm{VO}_{2}$ was calculated from the last 10 minutes of every activity each participant performed and were transformed into gross METs for each activity by dividing by 3.5. One kilogram was added to measured body weight for each participant to compensate for the added weight of the calorimetric unit and accelerometers worn by the individual. Consequently, the malfunctioning CSA resulted in the exclusion of 11 participants from the data analysis. For all activities, each participant wore two model 7164 CSA accelerometers, one on the right hip via belt and the other on the dominant hand via Velcro wrist strap. The CSA accelerometers were set to a 60-second epoch time interval. Linear regression analyses was used to predict METs from 1) only CSA hip counts 2), only CSA wrist counts, and 3) CSA hip and wrist counts for all activities performed.

Mean and standard deviation values for the CSA model 7164 accelerometers were calculated and the association between METs and CSA hip counts correlation ( $\mathrm{r}=0.563, \mathrm{P}<0.001)$. Cut points for 3 METs (light activity) was reported as 574, 6 METs (moderate activity) 4945 counts, and 9 METs (hard activity) 9317 counts were reported.

Nichols: Thirty male and 30 female volunteers, age 18 to 35 with participants instructed not to eat more than a light snack for 3 hours before testing. All 60 participants wore a Tritrac R3D activity monitor on each hip, each inside a pouch at hip level with the front edge at the mid-axillary line of each subject. Participants were then required to perform the following activities on a treadmill; horizontal walking and jogging at 3.2, 6.4 kilometers per hour, at 9.7 kilometers per hour, and walking at 6.4 kilometers per hour up a $5 \%$ grade on a motorized treadmill. These speeds were selected to correspond to MET levels within the range for light, moderate, and hard PA, according to guidelines in the Surgeon General's Report [13]. Each activity was performed for 5 minutes with a 1-minute rest interval between activities. The order of exercise bouts was randomized.

Energy expenditure (EE) was measured by indirect calorimetric using an automated metabolic cart and MET levels were calculated by dividing $\mathrm{VO}_{2}$ values (milliliters per kilogram per minute) by 3.5 ( $1 \mathrm{MET}$ ). The last 2 minutes of each activity were used for analysis, with means of the sum of the vector magnitudes and the total estimated kcal expended (predicted resting EE plus activity EE) in each specified time interval (epoch) for both the right and left hip. Reliability was assessed by comparing repeated measures on 1) 10 male and 10 female participants as well as 2) securely fastening to a mechanical shake table, which induced motion in three planes.

No gender differences were found and analyses were conducted on the entire sample. Linear regression analysis was used to assess the relationship between vector magnitude and energy expenditure and cut-off points for light, moderate, and vigorous PA were determined. The authors found a robust relationship between energy expenditure (kilocalories per kilogram per minute) with $\mathrm{R}^{2}=0.90$. The cut points corresponding to 2,4 , and 7 MET were 650,1772 , and 3455 counts, respectively. The second article utilized 30 of the original study participants, asking them to walked and jogged outdoors on a 400-m track. The association between CSA counts and both $\mathrm{VO}_{2}$ and velocity was linear in the field study, but there were statistically significant differences between laboratory and field measures of CSA counts for light and vigorous intensity with the conclusion that accelerometers may be used to quantify walking and jogging outdoors on level ground but laboratory equations may not be appropriate for use in field settings, particularly for light and vigorous activity. The field study cut points corresponding to 2 , 4, and 7 MET were 1576, 3285, and 5677 counts, respectively. These are not directly comparable to other studies because of the different MET values used to create cut points.

Brage: Twelve male subjects (age 22.7-30.0 years, mass 63.9-91.2 kilograms, height 169-199 centimeters) performed three treadmill trials (A, B, and C) and one field trial (F) with each trial were separated by at least 2 days.

Each trial consisted of treadmill ambulation of 5 minutes each with no rest in between at 3 and 6 kilometers per hour of walking and $8,9,10,12,14,16,18$, and 20 kilometers per hour of running until voluntary exhaustion. Mass-specific oxygen consumption rate $\left(\mathrm{VO}_{2}\right.$ per kilogram measured in milliliters per kilogram per minute) was measured continuously every 30 seconds (15-second sampling period, 15-second pause) during trials B and C, with expired air collected using a face mask and analyzed. The same protocol was used for the field trial but ended after 35 minutes, covering the speed range 3-14 kilometers per hour. Any incomplete intervals (duration $<5$ minutes) in trials $\mathrm{A}, \mathrm{B}$, and $\mathrm{C}$ were included in the analysis if their duration was $>=$ 2.5 minutes.

In all phases a total of four CSA units were worn, two CSA units on each hip, at the same position for all subjects and in all trials.

For each velocity, steady state $\mathrm{VO}_{2}$ per kilogram (trial $\mathrm{B}$ and $C$ only) and HR were calculated as the mean of the last 1.5 minutes of each velocity. In the final incomplete intervals, $\mathrm{VO}_{2}$ per kilogram and heart rate were expressed as the mean of the two highest values, respectively.

CSA counts per minute were calculated for each CSA unit and expressed as the mean of 4 minutes on each velocity in each trial, allowing 1 minute for speed change and/or adaptation. The mean of the means for all four accelerometers were also calculated.

The relationships between the outcome variables (speed or 
$\mathrm{VO}_{2}$ per kilogram) and the counts per minute were assessed with multiple linear regression modeling with adjustment for fitness and heart rate with all subjects completed the 3- to 14-kilometerper-hour intervals in all trials. Mean CSA outputs for each velocity in all four trials rose approximately linearly with speed until this relationship plateaus at 9 kilometers per hour with the CSA counts leveling at approximately 10,000 counts per minute. Cut points from this study suggest that light activity should be $<1810$, moderate activity $<5850$ and hard PA at $\geq 5850$.

Yngve: Fourteen male (mean age 23.7 2.6, mass 75.55 .5 kilograms, height 1.800 .05 meters) and 14 female (mean age 23.1 2.6, mass 62.03 .8 kilograms, height 1.690 .04 meters) volunteers participated in a laboratory study.

The test protocol included participants walking and running on an indoor track (105 meters) while wearing two accelerometers, one on the right hip (midaxillary line) and the other on the lower back (lumbar vertebrae 4-5). Participants were instructed to stand still for 5 minutes before beginning to walk at a "normal pace" and "fast pace" and to jog at a "comfortable pace" for 5 minutes at each stage in succession with a "brief stop" between each stage. These verbal anchors were independently interpreted by each study participant and were not quantified. The stages were timed and the walking and jogging speeds were calculated individually. The test was repeated on a motorized treadmill, using the individually calculated speeds from the track locomotion within 1 week. The same two activity monitors were used. A 15-second epoch was used with mean counts calculated from the $3^{\text {rd }}$ and $4^{\text {th }}$ minute of each stage. Mean Oxygen uptake $\left(\mathrm{VO}_{2}\right)$ was measured in 15-second intervals and calculated from the $3^{\text {rd }}$ and $4^{\text {th }}$ minute of each stage, including the standing stage. The metabolic energy turnover (MET) was calculated as one MET equal to a $\mathrm{VO}_{2}$ of 3.5 milliliters per kilogram per minute.

Linear regression models were created to assess the relationship between activity counts corresponding to moderate (3-5.99 METs) and vigorous (>=6 METs) for both track and treadmill tests. Cut points for the two locations for both the track and treadmill tests were similar, with the low back location being lower, with a slightly worse correlation value. Cut points for wearing the accelerometer on the right hip from this study suggest that light activity should be $<2631$ (track) or 2743 (treadmill), moderate activity $<6405$ (track) or 6583 (treadmill) and hard PA at $\geq 6405$ (track) or 6583 (tread mill).

Matthews: Matthews conducted a review of many articles comparing and contrasting the varying cut points [27]. Many articles utilized structured activities and most were only ambulatory activities (walking or running) and not free living activities. Matthews states that "in field-based studies, use of moderate cut points that are too low would be expected to result in an overestimation of time spent in moderate activity" (p. S516) [27]. Furthermore, he concludes that there is a definite relationship between increasing energy expenditure accelerometer counts however, it is not feasible to accurately state the threshold of physical inactivity from the current literature. Matthews further suggests that physical inactivity such as sitting and working quietly (e.g., reading, typing) rarely results in activity counts above 250 counts per minute and that the activities below 250 counts should be given a MET value of 1.0. Matthews also found that the highest cut point of 760 counts per minute provided the most accurate estimate of time spent in moderate intensity activity, although the individual variation around the mean was considerable. Matthews graphically described the activity count distributions across the 28 lightand moderate-intensity activities and notes this threshold will inappropriately capture some light-intensity activities and miss some moderate-intensity activities on an individual basis. Moreover, Matthews states that a single cut point for a waistmounted accelerometer will be unable to completely differentiate between all light- and moderate-intensity activities in free-living adults but the proposed count threshold would capture the majority of time spent in the activities at or above 3.6 METs.

\section{Statistics}

Correlation coefficients were calculated between cut points for all 78 participants. Correlation coefficients were between total minutes in light, moderate, and vigorous activity levels, and bouts of 10 and 20 minutes in moderate for each cut point. Sensitivity and specificity were calculated comparing each cut point with those proposed by Matthews et al. at each of the three levels of activity on a minute by minute basis to generate matrices for all comparisons. Sensitivity was calculated by dividing the total number minutes in agreement for that activity level (cell A) by the total number of minutes at that level as defined by the Matthews et al. cut points (cell A + cell C). Specificity was calculated by dividing the total number of minutes of agreement for not being in that activity level (cell D) by the total number of minutes in not at that activity level as defined by the Matthews et al. cut points (cell $\mathrm{B}+$ cell D). For example, on a minute by minute basis comparing light activity cut points between Swartz et al. and Matthews et al., the number in cell $A$ is the number of minutes where both sets of cut points agree that it was a minute of light activity. The number in cell B is the number of minutes where Swartz et al. had light activity level and Matthews et al. did not. Cell C was the converse; the total number of minutes that Matthews et al. counted in the light category but Swartz et al. did not. Cell D contains the total number of minutes where both cut points agree that the minute in question is not in the light category. These numbers can also be categorized by the overlap of the different cut points. For the example used above, in cell A, it would be the total number of minutes with counts between 574 and 760 , the overlap of the two light categories. Cell B would be the number of minutes between 760 and 4944, cell $C$ is the number of minutes between 251 and 574 , and cell $C$ is the sum of the total number of minutes below 251 or above 4944 . All statistics were calculated using SAS 9.1.3 (Cary, N.C, USA).

\section{Results}

Descriptive data for the population are found in Table 1. The mean age was 38.2 (standard deviation (SD) of 11.3) years, with a mean Body Mass Index (BMI) of 28.4 (SD 5.7) kg/m².

The cut points have markedly different mean values. Hendelman has the highest mean number of minutes in both 


\begin{tabular}{|c|c|c|c|}
\hline Variable & Category & $\mathbf{n}$ & $\begin{array}{l}\% \text { or Mean } \\
\text { (SD) }\end{array}$ \\
\hline \multirow[t]{2}{*}{ Gender } & Male & 50 & 73.5 \\
\hline & Female & 18 & 26.5 \\
\hline \multirow[t]{3}{*}{ Tobacco Use } & Never & 55 & 80.9 \\
\hline & Past Smoker & 11 & 16.2 \\
\hline & Yes, currently & 2 & 2.9 \\
\hline \multirow{4}{*}{$\begin{array}{l}\text { Quartiles of mean counts per } \\
\text { minute }\end{array}$} & $100-210 \mathrm{ct} / \mathrm{min}$ & 17 & 25.0 \\
\hline & $210-262 \mathrm{ct} / \mathrm{min}$ & 17 & 25.0 \\
\hline & $262-341 \mathrm{ct} / \mathrm{min}$ & 17 & 25.0 \\
\hline & $341-650 \mathrm{ct} / \mathrm{min}$ & 17 & 25.0 \\
\hline \multirow{3}{*}{$\begin{array}{l}\text { Tertiles of mean counts per } \\
\text { minute }\end{array}$} & $\begin{array}{c}100-231.5 \mathrm{ct} / \\
\min \end{array}$ & 22 & 32.4 \\
\hline & $\begin{array}{l}231.5-314 \mathrm{ct} / \\
\min \end{array}$ & 23 & 33.8 \\
\hline & $314-650 \mathrm{ct} / \mathrm{min}$ & 23 & 33.8 \\
\hline \multirow{2}{*}{$\begin{array}{l}\text { Ever seen a Health Care } \\
\text { Provider for your Low Back } \\
\text { Pain }\end{array}$} & No & 42 & 61.8 \\
\hline & Yes & 26 & 38.2 \\
\hline \multirow{4}{*}{$\begin{array}{l}\text { How often do you feel down, } \\
\text { blue, or depressed? }\end{array}$} & Never & 20 & 29.4 \\
\hline & Seldom & 38 & 55.9 \\
\hline & Often & 10 & 14.7 \\
\hline & Always & 0 & 0.0 \\
\hline \multirow[t]{2}{*}{ Low Back Pain Incident Case } & No & 38 & 55.9 \\
\hline & Yes & 30 & 44.1 \\
\hline Age (years) & & 68 & $38.23(11.3)$ \\
\hline Body Mass Index (kg/m2) & & 68 & $28.37(5.7)$ \\
\hline \multicolumn{4}{|l|}{$\begin{array}{l}\text { Quartiles of mean counts per } \\
\text { minute }\end{array}$} \\
\hline 1 (Lowest, 0-25\%) & & 17 & $178.43(25.1)$ \\
\hline $2(25-50.0 \%)$ & & 17 & $238.88(11.9)$ \\
\hline $3(50-75 \%)$ & & 17 & $303.53(20.4)$ \\
\hline 4 (Highest, 75-100\%) & & 17 & $443.42(88.1)$ \\
\hline \multicolumn{4}{|l|}{$\begin{array}{l}\text { Tertiles of mean counts per } \\
\text { minute }\end{array}$} \\
\hline Lowest tertile & & 22 & $189.4(30.3)$ \\
\hline Middle tertile & & 23 & $266.6(26.7)$ \\
\hline Highest tertile & & 23 & $412.7(92.0)$ \\
\hline
\end{tabular}

Table 1: Demographic Data.

the light and moderate category, reflecting the lowest cut point for light activity and the broadest range for moderate activity. Conversely, the cut points proposed by Freedson et al. have the lowest number of mean minutes in light activity at 253.76, followed closely by the cut points proposed by Swartz et al. at 266.35. The cut points proposed by Swartz et al. for both moderate and vigorous exercise have the smallest mean values, reflecting the fact that these are the highest values for cut points for these two activity levels [Table 2]. The total mean and standard deviation for total values demonstrate that there is the most consistency between cut points at the vigorous activity level, and the least consistency at the moderate activity level.

Evaluating only those cut points derived from structured activities, there is much more consistency in this population, with vigorous activity having the smallest proportional standard deviation and light activity having the highest proportional standard deviation. Investigating only those cut points derived from free-living activity, there are much higher standard deviations, with the lowest being for light PA, and the highest being for vigorous activity [Table 3].

Results in Table 4 demonstrate that there are strong correlations between many of the measures for light activity (generally described as 1-3 METs). Most notably, there are several correlation coefficients above 0.90. These strongly positive correlations are between Brage and Crouter, Nichols and Freedson, Swartz and Freedson, and Yngve and both Brage and Crouter. Of these five strong correlations, three were between laboratory derived and free-living derived cut points, and two were between laboratory derived cut points only. There were no strong relationships between free-living derived cut points only. There are also many correlation coefficients with low values (below 0.25 ) and one with a slightly negative value. These poorly correlated measures are between Nichols and Crouter, Hendelman and Freedson, Hendelman and Nichols, Hendelman and Swartz. Of the four poorly correlated measures, three were between free-living derived and laboratory derived cut points, and one was between free-living derived cut points only.

Results in Table 5 demonstrate that there are strong correlations between many of the measures for moderate activity (generally described as 3-6 METs). Most notably, there are several correlation coefficients above 0.90 . These strongly positive correlations are between Brage and Freedson, Brage and Yngve, Freedson and Yngve, and Nichols and Yngve. All four of these strong correlations were between laboratory derived cut points only. There were no strong relationships between free-

\begin{tabular}{|c|c|c|c|c|c|}
\hline & $\begin{array}{c}\text { Free- } \\
\text { living or } \\
\text { Structured } \\
\text { Activity }\end{array}$ & Inactive & Light & Moderate & Vigorous \\
\hline Brage et al. & $\begin{array}{c}\text { Structured } \\
\text { (Labora- } \\
\text { tory) }\end{array}$ & $<1$ & 1-1809 & $1810-5850$ & $>5850$ \\
\hline $\begin{array}{c}\text { Crouter et } \\
\text { al.* }\end{array}$ & Free-Living & $<50$ & $50-760$ & & \\
\hline $\begin{array}{l}\text { Freedson } \\
\text { et al. }\end{array}$ & $\begin{array}{l}\text { Structured } \\
\text { (Labora- } \\
\text { tory) }\end{array}$ & $<1258$ & $1258-1950$ & $1951-5724$ & $>5724$ \\
\hline $\begin{array}{l}\text { Hendelman } \\
\text { et al.* }\end{array}$ & Free-Living & $<1$ & $1-190$ & $191-7525$ & $>7525$ \\
\hline $\begin{array}{c}\text { Matthews } \\
\text { et al.* }\end{array}$ & Free-Living & $<251$ & $251-760$ & $761-5724$ & $>5724$ \\
\hline $\begin{array}{c}\text { Nichols et } \\
\text { al. }^{\dagger}\end{array}$ & $\begin{array}{c}\text { Structured } \\
\text { (Track) }\end{array}$ & $<1576$ & $1576-3284$ & $3285-5677$ & $>5676$ \\
\hline $\begin{array}{c}\text { Swartz et } \\
\text { al. }^{*}\end{array}$ & Free-Living & $<574$ & $574-4944$ & $4945-9316$ & $>9316$ \\
\hline Yngve et al. & $\begin{array}{c}\text { Structured } \\
\text { (Track) }\end{array}$ & $<1$ & $1-2631$ & $2632-6404$ & $>6404$ \\
\hline
\end{tabular}

${ }^{\dagger}$ Utilized different MET cut points of 2, 4, and 7 Mets, respectively.

Table 2: Type of Activity and Corresponding Cut points Used for Each Activity Level. 


\begin{tabular}{|c|c|c|c|c|c|c|}
\hline & & \multicolumn{5}{|c|}{ Activity Level } \\
\hline & & Light & Moderate & Vigorous & $\begin{array}{l}10 \text { Minute } \\
\text { Moderate Bout }\end{array}$ & $\begin{array}{l}20 \text { Minute } \\
\text { Moderate Bout }\end{array}$ \\
\hline \multirow{8}{*}{ 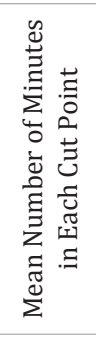 } & Brage et al. & 3817.46 & 240.24 & 2.26 & 1.92 & 0.62 \\
\hline & Crouter et al.* & 2190.24 & & & & \\
\hline & Freedson et al. & 253.76 & 204.49 & 2.59 & 1.59 & 0.51 \\
\hline & Hendelman et al.* & 3959.26 & 2116.55 & 0.59 & 54.29 & 19.44 \\
\hline & Matthews et al.* & 1942.69 & 861.33 & 2.59 & 12.74 & 3.60 \\
\hline & Nichols et al. ${ }^{\dagger}$ & 1017.58 & 46.27 & 2.74 & 0.37 & 0.08 \\
\hline & Swartz et al. * & 266.35 & 6.67 & 0.19 & 0.08 & 0.04 \\
\hline & Yngve et al. & 1112.59 & 99.08 & 1.38 & 0.74 & 0.26 \\
\hline \multirow{2}{*}{ 퓸 } & Mean & 1819.99 & 510.66 & 1.76 & 10.25 & 3.51 \\
\hline & Standard Deviation & 1450.60 & 764.61 & 1.05 & 19.92 & 7.13 \\
\hline \multirow{2}{*}{ 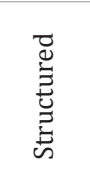 } & Mean & 1550.35 & 147.52 & 2.24 & 1.16 & 0.37 \\
\hline & Standard Deviation & 1559.53 & 90.26 & 0.61 & 0.72 & 0.24 \\
\hline \multirow{2}{*}{ 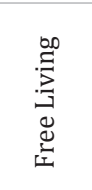 } & Mean & 2089.63 & 994.85 & 1.12 & 22.37 & 7.69 \\
\hline & Standard Deviation & 1511.25 & 1061.26 & 1.28 & 28.36 & 10.33 \\
\hline
\end{tabular}

Table 3: Mean Number of Minutes in Each Level of Activity and Cut Point and in Total.

\begin{tabular}{|c|c|c|c|c|c|c|c|c|}
\hline & 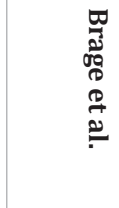 & 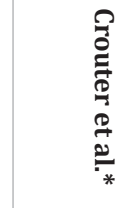 & $\begin{array}{l}7 \\
7 \\
0 \\
0 \\
0 \\
0 \\
0 \\
0 \\
2 \\
2\end{array}$ & 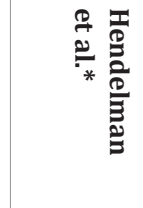 & 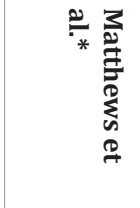 & $\begin{array}{l}z \\
\frac{2}{2} \\
\frac{0}{4} \\
0 \\
0 \\
0\end{array}$ & 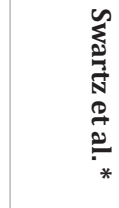 & 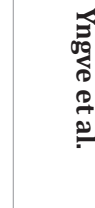 \\
\hline Brage et al. & 1.000 & & & & & & & \\
\hline Crouter et al.* & 0.941 & 1.000 & & & & & & \\
\hline Freedson et al. & 0.503 & 0.284 & 1.000 & & & & & \\
\hline Hendelman et al.* & 0.762 & 0.732 & -0.010 & 1.000 & & & & \\
\hline Matthews et al.* & 0.866 & 0.899 & 0.478 & 0.415 & 1.000 & & & \\
\hline Nichols et al. ${ }^{\dagger}$ & 0.437 & 0.219 & 0.908 & 0.037 & 0.363 & 1.000 & & \\
\hline Swartz et al. * & 0.611 & 0.417 & 0.958 & 0.065 & 0.622 & 0.905 & 1.000 & \\
\hline Yngve et al. & 0.996 & 0.919 & 0.567 & 0.731 & 0.861 & 0.514 & 0.670 & 1.00 \\
\hline
\end{tabular}

Table 4: Pearson Correlation Coefficients for Total Minutes of Light Activity.

living derived cut points only. There was also one correlation coefficient below 0.25; the measure between Hendelman and Swartz which was between free-living derived cut points only.

Results in Table 6 demonstrate that there are strong correlations between many of the measures for vigorous activity (generally described as 6 or more METs). Most notably, there are several correlation coefficients above 0.90. Freedson and Matthews utilize the same cut point for vigorous activity. These strongly positive correlations are between Brage and Freedson, Matthews, Nichols, and Yngve, Freedson and both
Nichols and Yngve, Hendelman and Yngve, Matthews and Yngve, and Nichols and Yngve. Of these 10 strong correlations, 6 were between laboratory derived cut points only and 4 were between laboratory derived and free-living derived cut points. There were no strong relationships between free-living derived cut points only or correlation coefficients with low values (below 0.25 ).

Correlation coefficients between cut points for combined moderate or vigorous activity levels (data not shown) were not markedly different from moderate activity alone, likely due to few minutes in the vigorous category. 


\begin{tabular}{|c|c|c|c|c|c|c|c|}
\hline & 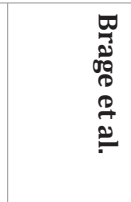 & 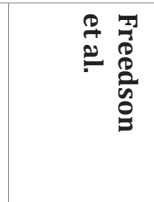 & 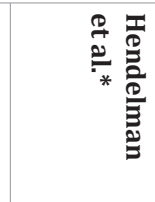 & 華 & 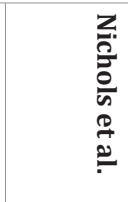 & 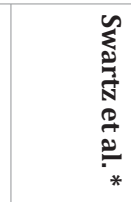 & 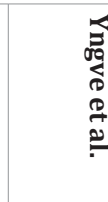 \\
\hline Brage et al. & 1.000 & & & & & & \\
\hline Freedson et al. & 0.996 & 1.000 & & & & & \\
\hline Hendelman et al.* & 0.632 & 0.589 & 1.000 & & & & \\
\hline Matthews et al.* & 0.834 & 0.791 & 0.883 & 1.000 & & & \\
\hline Nichols et al. ${ }^{\dagger}$ & 0.841 & 0.874 & 0.291 & 0.492 & 1.000 & & \\
\hline Swartz et al. * & 0.528 & 0.549 & 0.119 & 0.262 & 0.717 & 1.000 & \\
\hline Yngve et al. & 0.933 & 0.957 & 0.418 & 0.624 & 0.966 & 0.647 & 1.000 \\
\hline
\end{tabular}

Table 5: Correlation Coefficients for Total Minutes of Moderate Activity.

\begin{tabular}{|c|c|c|c|c|c|c|c|}
\hline & 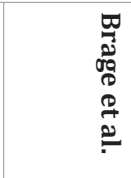 & 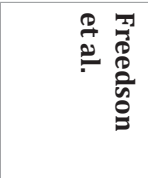 & 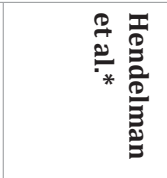 & 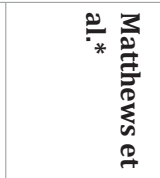 & 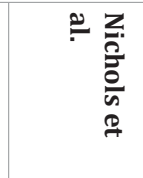 & 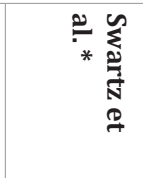 & 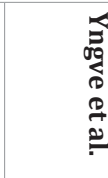 \\
\hline Brage et al. & 1.000 & & & & & & \\
\hline Freedson et al. & 0.996 & 1.000 & & & & & \\
\hline Hendelman et al.* & 0.741 & 0.708 & 1.000 & & & & \\
\hline Matthews et al.* & 0.996 & 1.000 & 0.708 & 1.000 & & & \\
\hline Nichols et al. ${ }^{\dagger}$ & 0.996 & 0.999 & 0.720 & 0.999 & 1.000 & & \\
\hline Swartz et al. * & 0.589 & 0.585 & 0.476 & 0.585 & 0.588 & 1.000 & \\
\hline Yngve et al. & 0.942 & 0.921 & 0.905 & 0.921 & 0.927 & 0.534 & 1.000 \\
\hline
\end{tabular}

Table 6: Pearson Correlation Coefficients for Total Minutes of Vigorous Activity.

\begin{tabular}{|c|c|c|c|c|c|c|c|}
\hline & 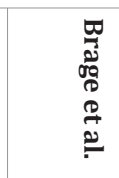 & 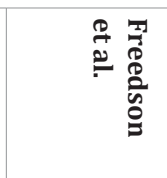 & 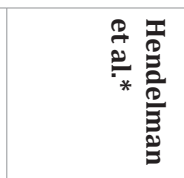 & 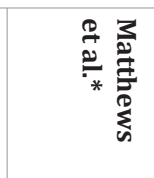 & 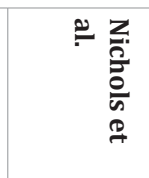 & 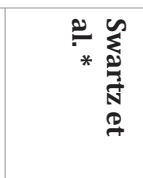 & 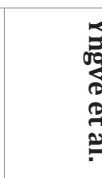 \\
\hline Brage et al. & 1.000 & & & & & & \\
\hline Freedson et al. & 0.976 & 1.000 & & & & & \\
\hline Hendelman et al.* & 0.256 & 0.227 & 1.000 & & & & \\
\hline Matthews et al.* & 0.549 & 0.479 & 0.540 & 1.000 & & & \\
\hline Nichols et al. ${ }^{\dagger}$ & 0.738 & 0.774 & 0.004 & 0.260 & 1.000 & & \\
\hline Swartz et al. * & 0.209 & 0.206 & -0.024 & 0.041 & 0.257 & 1.000 & \\
\hline Yngve et al. & 0.811 & 0.857 & 0.022 & 0.256 & 0.935 & 0.387 & 1.000 \\
\hline
\end{tabular}

Table 7: Pearson Correlation Coefficients for 10 Minute Bouts of Moderate Activity. 
Results in Table 7 demonstrate that there are still strong correlations between many of the measures for 10 minute bouts of moderate activity (generally described as 3-6 METs). Most notably, there are several correlation coefficients above 0.90 . These strongly positive correlations are between 1) Brage and Freedson and 2) Nichols and Yngve. Both of these strong correlations were between laboratory derived cut points only. There were no strong relationships between free-living derived cut points. There were several poorly correlated measures of 10-minute bouts of activity as defined by correlation coefficients below 0.25 . These poorly correlated measures are those between 1) Brage and Swartz, 2) Freedson and Hendelman, 3) Freedson and Swartz, 4) Hendelman and Nichols, 5) Hendelman and Swartz, 6) Hendelman and Yngve, and 7) Matthews and Swartz. Of these seven poor correlations, six were between laboratory and free-living derived cut points, while one (Matthews and Swartz) is between two free-living derived cut points.

Results in Table 8 demonstrate that there are still strong correlations between many of the measures for 20-minute bouts of moderate activity (generally described as 3-6 METs). Most notably, there are several correlation coefficients above 0.90 . These strongly positive correlations are between 1) Brage and Freedson and 2) Freedson and Yngve. Both of these strong correlations were between laboratory derived cut points only. There were no strong relationships between free-living derived cut points. There were several poorly correlated measures of 20 -minute bouts of activity as defined by correlation coefficients below 0.25 . These poorly correlated measures are those between 1) Brage and Swartz, 2) Freedson and Swartz, 3) Hendelman and Nichols, 4) Hendelman and Swartz, 5) Hendelman and Yngve, 6) Matthews and Nichols, and 7) Matthews and Swartz. Of these seven poor correlations, six were between laboratory and freeliving derived cut points, while one (Matthews and Swartz) is between two free-living derived cut points.

Sensitivity and specificity was also calculated, comparing each set of cut points to the Matthews et al. cut points (Table 9). For light activity sensitivities were generally dichotomized and ranged from 1.000 for Crouter et al., Brage et al. and Yngve et al. to 0.000 for Freedson et al., Hendelman et al. and Nichols et al. Only Swartz et al. had a sensitivity that was between 1.000 and 0.000 , with a value of 0.249 . Specificity for light activity ranged from 0.033 for Yngve et al. to 0.917 for Freedson et al., with four of the comparisons being above 0.500 and three being below 0.500 . Sensitivity for moderate activity had a large range as well, with low values of 0.005 for Swartz et al. and 0.054 for Nichols et al. to 1.000 for Hendelman et al. Only the Hendelman et al. sensitivity for moderate activity was above 0.500 . Specificity for moderate activity levels was generally higher as compared to light activity, with values ranging from 0.609 for Hendelman et al. to 1.000 for Brage et al., Freedson et al., Nichols et al. and Yngve et al. All specificity values for moderate activity were above 0.500 . Sensitivity for minutes of vigorous activity were similarly high with only two having values below 0.500 , Swartz et al. and Hendelman et al. with sensitivities of 0.067 and 0.245 , respectively. There were two cut points, Freedson et al. and Nichols et al., with sensitivities of 1.000. All of but one of the specificity values for vigorous activity was equal to 1.000 , with the only exception being Nichols et al. with a value of 0.99995 .

\section{Discussion}

There is a broad spectrum of total minutes spent in each activity level, as defined by the various cut points. The mean number of total minutes in each activity level as proposed by each author vary significantly. It appears that there are a large proportion of participants in this free-living study who have low total minute counts, many of which would be considered inactive by the relatively high minimum threshold for light activity proposed by several authors. Conversely, there are few minutes of vigorous activity, independent on what cut point is chosen for this category.

Hendelman et al. propose both the smallest range and lowest cut point for light PA, between 1 and 190 counts per minute, but has the widest range for moderate activity, between 191 and 7525 counts per minute, has the largest mean number of minutes in each category. The cut points proposed by Swartz et al. have the highest minimum value for light activity of any of the free-living cut points, but the largest range for light activity, has the lowest mean number of minutes in that category. Both of

\begin{tabular}{|c|c|c|c|c|c|c|c|}
\hline & 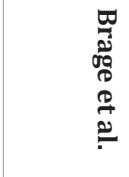 & 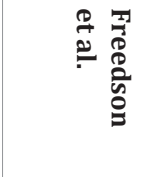 & 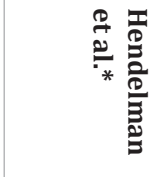 & • & 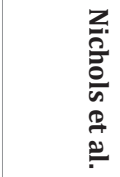 & 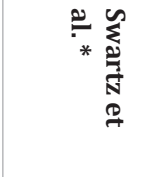 & 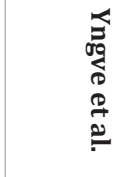 \\
\hline Brage et al. & 1.000 & & & & & & \\
\hline Freedson et al. & 0.960 & 1.000 & & & & & \\
\hline Matthews et al.* & 0.521 & 0.512 & 0.579 & 1.000 & & & \\
\hline Nichols et al. ${ }^{\dagger}$ & 0.539 & 0.615 & 0.066 & 0.189 & 1.000 & & \\
\hline Swartz et al. * & 0.1422 & 0.182 & -0.048 & -0.010 & 0.594 & 1.000 & \\
\hline Yngve et al. & 0.855 & 0.901 & 0.163 & 0.367 & 0.760 & 0.336 & 1.000 \\
\hline
\end{tabular}

Table 8: Pearson Correlation Coefficients for 20 Minute Bouts of Moderate Activity. 


\begin{tabular}{|c|c|c|c|}
\hline & Cut Point & Sensitivity & Specificity \\
\hline \multirow{7}{*}{ Light } & Crouter et al.* & 1.000 & 0.615 \\
\hline & Brage et al. & 1.000 & 0.080 \\
\hline & Freedson et al. & 0.000 & 0.917 \\
\hline & Hendelman et al.* & 0.000 & 0.360 \\
\hline & Nichols et al. $^{\dagger}$ & 0.000 & 0.912 \\
\hline & Swartz et al. * & 0.249 & 0.719 \\
\hline & Yngve et al. & 1.000 & 0.033 \\
\hline \multirow{6}{*}{ Moderate } & Brage et al. & 0.280 & 1.000 \\
\hline & Freedson et al. & 0.240 & 1.000 \\
\hline & Hendelman et al.* & 1.000 & 0.609 \\
\hline & Nichols et al. ${ }^{\dagger}$ & 0.054 & 1.000 \\
\hline & Swartz et al. * & 0.005 & 0.999 \\
\hline & Yngve et al. & 0.115 & 1.000 \\
\hline \multirow{6}{*}{ Vigorous } & Brage et al. & 0.894 & 1.000 \\
\hline & Freedson et al. & 1.000 & 1.000 \\
\hline & Hendelman et al.* & 0.245 & 1.000 \\
\hline & Nichols et al. ${ }^{\dagger}$ & 1.000 & 0.99995 \\
\hline & Swartz et al. * & 0.067 & 1.000 \\
\hline & Yngve et al. & 0.558 & 1.000 \\
\hline
\end{tabular}

Table 9: Sensitivity and Specificity for Cut Points Compared to Matthews et al.* for Light, Moderate, and Vigorous Minutes of Activity.

these sets of cut points were derived from measures free-living physical activities. The mean and standard deviations for the structured activity derived cut points are generally lower than those for the free-living activity derived cut points. The highest consistency is for vigorous PA as defined by structured activity cut points followed by moderate PA also defined by structured activity cut points. Conversely, the least consistent measures are for vigorous PA as defined by free-living activity cut points followed by moderate PA also defined by free-living activity cut points. These inconsistencies are largely driven by the mean total minutes for the Hendelman et al. cut points. These differences in cut points have demonstrated markedly different mean values for each activity level.

Correlations between measures infer similar findings. For light activity levels, the highest correlations were between the six structured activity and structured activity cut point pairs and the lowest were between the six free-living activity and free-living activity cut point pairs, with a mean correlation coefficients of 0.6541 and 0.5250 , respectively. The mean of the correlation coefficients between the 16 free-living activity and structured activity pairs was 0.5995 . Moderate activity levels demonstrate more pronounced differences. For the six moderate structured activity pairs, the mean of the correlation coefficients was 0.9278 , as compared to 0.4211 for the three moderate free-living correlation pairs. Again the mean of the correlation coefficients for the structured activity and free-living activity pairs was between the two, with a value of 0.5926 . For vigorous activity, means of the correlation coefficients were higher for structured activity pairs, free-living pairs, and mixed activity type pairs, with mean values of $0.9634,0.5898$, and 0.7739 respectively. These data should be taken lightly as the cut points for Matthews and Freedson are the same and there are few minutes at these levels. Continuous bouts of moderate activity demonstrate the most significant difference between structured activity and free-living activity. Mean values for correlation coefficients for 10-minute bouts of moderate activity were again highest for structured activity pairs and lowest for free-living pairs, 0.8486 and 0.1859 , respectively. Mean values for bouts of 20 minutes were highest for structured activity pairs and lowest for free-living pairs, 0.7716 and 0.1737 , respectively.

Sensitivity and specificity findings show a broad range, indicative of broad range and degree of overlap between different cut points. There was little agreement when looking at sensitivity and specificity values between free-living or laboratory defined cut points. There were many sensitivity and specificity measures, particularly in the vigorous activity level, that had values of 1.000 indicating complete agreement. This is not surprising when compared with the cut point values. The cut points for light activity for Matthews et al. are completely encapsulated by the cut points proposed by Brage et al., Crouter et al., and Yngve et al., yielding very high sensitivities. Conversely, the Brage et al. and Yngve et al. cut points also include much higher counts per minute than the 760 upper cut point of Matthews et al., 1809 and 2631, respectively. This broad inclusion gives a very low specificity value when comparing light activity by Brage et al. or Yngve et al. with Matthews et al. Similar relationships can be found in both directions when looking at sensitivity and specificity values in the light and moderate activity categories. Because there were relatively few minutes of vigorous activity by any of the cut points, sensitivity and specificity values for this activity level are generally stronger, indicating that in this population at the highest levels of activity, the different cut points may track well as compared to the Matthews et al. cut points. However, due to the small number of minutes in this activity level, these sensitivities and specificities must be interpreted cautiously.

This study has many strengths. This study included a relatively large number of participants. All participants wore the accelerometer for at least 12 hours for at least 5 days, one of which was a weekend day. All activity levels were reported as being normal for each participant. Participants completed an activity diary and a 7-day PA Questionnaire, which was consistent with accelerometer results. All participants were enrolled within 82 days, and meteorological data at that time were consistent.

This study could have been further improved by measuring maximum $\mathrm{VO}_{2}$ in all or some of the population to ascertain accuracy of the cut points for this occupational population.

\section{Conclusions}

These findings suggest that cut points based on structured activities are the most congruous; however they may not be the most accurate. Significant differences between cut points exist, particularly those derived from free-living data, which is possibly due to the variable nature of the activity. These differences could dramatically affect findings relating outcomes to these different measures of activity. Care should be taken when choosing cut 
points for research to correctly represent the population and activity type of interest.

\section{Authors Contributions}

MST conceived of the project, collected and analyzed the data, and was primarily responsible for writing the manuscript. KTH assisted in writing the manuscript and refining the project. TKB assisted in refining the project, data analysis and writing the manuscript. AG assisted in writing the manuscript and refining the project. $\mathrm{CP}$ assisted in writing the manuscript and refining the project.

\section{Acknowledgements}

This study has been funded, in part, by grants from the National Institute for Occupational Safety and Health (NIOSH/ CDC), Education and Research Center training grant T42/ CCT810426-10. The CDC/NIOSH is not involved in the study design, data analyses or interpretation of the data. No additional external funding received for this study.

The authors wish to acknowledge the contributions of numerous individuals, many of whom perform volunteer, or only partially compensated work on this project. Additional sources of funding include the Universities and other, non-commercial resources.

\section{References}

1. Karmisholt, K., \& Gotzsche, P.C. (2005). Physical activity for secondary prevention of disease. Systematic reviews of randomised clinical trials. Dan Med Bull, 52(2), 90-94.

2. Klaber Moffett, J.A., Carr, J., \& Howarth, E. (2004). High fear-avoiders of physical activity benefit from an exercise program for patients with back pain. Spine, 29(11), 1167-1172.

3. Staal, J.B., Hlobil, H., van Tulder, M.W., Koke, A.J., Smid, T., \& van Mechelen, W. (2002). Return-to-work interventions for low back pain: a descriptive review of contents and concepts of working mechanisms. Sports Med, 32(4), 251-267.

4. Andersen, L.B., Schnohr, P., Schroll, M., \& Hein, H.O. (2000). All-cause mortality associated with physical activity during leisure time, work, sports, and cycling to work. Arch Intern Med, 160(11), 1621-1628.

5. Berlin, J.A., \& Colditz, G.A. (1990). A meta-analysis of physical activity in the prevention of coronary heart disease. Am J Epidemiol, 132(4), 612-628.

6. Blair, S.N., Kohl, H.W., Barlow, C.E., Paffenbarger, R.S., Gibbons, L.W., \& Macera, C.A. (1995). Changes in physical fitness and all-cause mortality. A prospective study of healthy and unhealthy men. JAMA. 273(14), 1093-1098.

7. Cerhan, J.R., Torner, J.C., Lynch, C.F., Rubenstein, L.M., Lemke, J.H., Cohen, M.B., et al. (1997). Association of smoking, body mass, and physical activity with risk of prostate cancer in the Iowa 65+ Rural Health Study (United States). Cancer Causes Control, 8(2), 229-238.

8. Colditz, G.A., Feskanich, D., Chen, W.Y., Hunter, D.J., \& Willett, W.C. (2003). Physical activity and risk of breast cancer in premenopausal women. Br J Cancer, 89(5), 847-851.

9. Powell, K.E., Thompson, P.D., Caspersen, C.J., \& Kendrick, J.S. (1987). Physical activity and the incidence of coronary heart disease. Annu Rev Public Health. 8, 253-287.
10. Van Gaal, L.F., Mertens, I.L., \& De Block, C.E. (2006). Mechanisms linking obesity with cardiovascular disease. Nature, 444(7121), 875-880.

11.Porter, R.W., Adams, M.A., \& Hutton, W.C. (1989). Physical activity and the strength of the lumbar spine. Spine, 14(2), 201-203.

12. Sternfeld, B., Ainsworth, B.E., \& Quesenberry, C.P. (1999). Physical activity patterns in a diverse population of women. Prev Med, 28(3), 313-323.

13. Steptoe, A., Roy, M.P., Evans, O., \& Snashall, D. (1995). Cardiovascular stress reactivity and job strain as determinants of ambulatory blood pressure at work. J Hypertens, 13(2), 201-210.

14. Haskell, W.L., Lee, I.M., Pate, R.R., Powell, K.E., Blair, S.N., Franklin, B.A., et al. (2007). Physical activity and public health: updated recommendation for adults from the American College of Sports Medicine and the American Heart Association. Med Sci Sports Exerc, 39(8), 1423-1434.

15. Nelson, M.E., Rejeski, W.J., Blair, S.N., Duncan, P.W., Judge, J.O., King, A.C., et al. (2007). Physical activity and public health in older adults: recommendation from the American College of Sports Medicine and the American Heart Association. Med Sci Sports Exerc, 39(8), 14351445.

16. Bassett, D.R. (2000). Validity and reliability issues in objective monitoring of physical activity. Res Q Exerc Sport, 71, S30-36.

17. Ham, S.A., Reis, J.P., Strath, S.J., Dubose, K.D., \& Ainsworth, B.E. (2007). Discrepancies between methods of identifying objectively determined physical activity. Med Sci Sports Exerc, 39(1), 52-58.

18. Freedson, P.S., Melanson, E., \& Sirard, J. (1998). Calibration of the computer science and applications, Inc. accelerometer. Med Sci Sports Exerc, 30(5), 777-781.

19. Hendelman, D., Miller, K., Baggett, C., Debold, E., \& Freedson, P. (2000). Validity of accelerometry for the assessment of moderate intensity physical activity in the field. Med Sci Sports Exerc, 32, S442-449.

20.Swartz, A.M., Strath, S.J., Bassett, D.R., O’Brien, W.L., King, G.A., \& Ainsworth, B.E. (2000). Estimation of energy expenditure using CSA accelerometers at hip and wrist sites. Med Sci Sports Exerc, 32, S450456.

21.Leenders, N.Y., Nelson, T.E., \& Sherman, W.M. (2003) Ability of different physical activity monitors to detect movement during treadmill walking. Int J Sports Med, 24(1), 43-50.

22. Nichols, J.F., Morgan, C.G., Sarkin, J.A., Sallis, J.F., \& Calfas, K.J. (1999). Validity, reliability, and calibration of the Tritrac accelerometer as a measure of physical activity. Med Sci Sports Exerc, 31(6), 908-912.

23. Nichols, J.F., Morgan, C.G., Chabot, L.E., Sallis, J.F., \& Calfas, K.J. (2000). Assessment of physical activity with the computer science and applications, Inc., accelerometer: laboratory versus field validation. Res Q Exerc Sport, 71(1), 36-43.

24. Brage, S., Wedderkopp, N., Franks, P.W., Andersen, L.B., \& Froberg, K. (2003). Reexamination of validity and reliability of the CSA monitor in walking and running. Med Sci Sports Exerc, 35(8), 1447-1454.

25. Yngve, A., Nilsson, A., Sjostrom, M., \& Ekelund, U. (2003). Effect of monitor placement and of activity setting on the MTI accelerometer output. Med Sci Sports Exerc, 35(2), 320-326.

26. Crouter, S.E., Clowers, K.G., \& Bassett, D.R. (2006). A novel method for using accelerometer data to predict energy expenditure. J Appl Physiol, 100(4), 1324-1331.

27. Matthews, C.E. (2005). Calibration of accelerometer output for adults. Med Sci Sports Exerc, 37, S512-522. 
28. Garg A, Hegmann KT, Moore JS, Kapellusch J, Thiese MS, et al. (2013) Study protocol title: a prospective cohort study of low back pain. BMC Musculoskelet Disord 14: 84.

29. Thiese MS, Hegmann KT, Garg A, Porucznik C, Behrens T (2011) The predictive relationship of physical activity on the incidence of low back pain in an occupational cohort. J Occup Environ Medicine Apr 53: 364-371.

30. Thiese MS, Hegmann KT, Wood EM, Garg A, Moore JS, et al. (2013) Low Back Pain Ratings for Lifetime, 1-Month Period, and Point Prevalences in a Large Occupational Population. Human Factors: The Journal of the Human Factors and Ergonomics Society.
31. Trost, S.G., McIver, K.L., \& Pate, R.R. (2005). Conducting accelerometerbased activity assessments in field-based research. Med Sci Sports Exerc, 37, S531-543.

32. Matthews, C.E., Ainsworth, B.E., Thompson, R.W., \& Bassett, D.R., (2002). Sources of variance in daily physical activity levels as measured by an accelerometer. Med Sci Sports Exerc, 34(8), 13761381.

33. Sallis, J.F., Haskell, W.L., Wood, P.D., Fortmann, S.P., Rogers, T., Blair, S.N., et al. (1985). Physical activity assessment methodology in the Five-City Project. Am J Epidemiol, 121(1), 91-106. 